\title{
Enhancing Decision-making Systems with Relevant Patient Information by Leveraging Clinical Notes
}

\author{
João Rafael Almeida ${ }^{1,2, *} \mathbb{C}^{\mathrm{a}}$, João Figueira Silva ${ }^{1, *} \mathbb{C}^{\mathrm{b}}$, Alejandro Pazos Sierra ${ }^{2} \mathbb{C}^{\mathrm{c}}$, Sergio Matos ${ }^{1} \mathbb{C}^{\mathrm{d}}$ \\ and José Luís Oliveira ${ }^{1}$ (D) \\ ${ }^{1}$ DETI/IEETA, University of Aveiro, Aveiro, Portugal \\ ${ }^{2}$ Department of Information and Communications Technologies, University of A Coruña, A Coruña, Spain
}

Keywords: $\quad$ EHR, CDSS, NLP, Clinical Notes, Clinical Decision-making, Treatment Guidance.

\begin{abstract}
Hospitalised patients suffering from secondary illnesses that require daily medication typically need personalised treatment. Although clinical guidelines were designed considering those circumstances, existing decision-support features fail in assimilating detailed relevant patient information, which opens up opportunities for systems capable of performing a real-time evaluation of such data against existing knowledge and providing recommendations during clinical treatments. In this paper, we present a proposal for a new feature to integrate with electronic health record (EHR) systems that enriches the health treatment process by automatically extracting information from patient medical notes and aggregating it in clinical protocols. Our goal is to leverage the historical component of the patient trajectory to improve clinical decision support systems performance.
\end{abstract}

\section{INTRODUCTION}

Progress in technology has proved fruitful for the field of medicine and health care throughout the years, leading to an enhanced quality of life for the general population. Tools and data sources originated from the merging of these two fields have fostered improvements in disease prevention, diagnosis and treatment, and can play an important role in clinical pipelines by assisting physicians in tasks such as clinical decision making and patient follow-up. Furthermore, by providing access to increasing amounts of medical data, it is possible to shift towards the more patient-centric view of personalised medicine.

Aside from scale, technology also brought diversity to medical data, comprising various data types such as medical imaging, genomic, signal, or laboratory data, which must be stored and organised. Electronic health records (EHRs) provide electronic support to agglutinate administrative and medical data

\footnotetext{
a (i) https://orcid.org/0000-0003-0729-2264

b (iD https://orcid.org/0000-0001-5535-754X

c (10) https://orcid.org/0000-0003-2324-238X

d (1D https://orcid.org/0000-0003-1941-3983

e (iD https://orcid.org/0000-0002-6672-6176

${ }^{*}$ Both authors contributed equally to this work.
}

from various sources and to centralise information at the patient level (Katehakis and Tsiknakis, 2006; Costa, 2004), enabling the documentation of a patient's health status throughout time and representing the patient trajectory. By having a longitudinal view of the patient medical history accessible in a single structure, the EHR can provide physicians with important contextual information therefore rendering EHRs as an important piece to support the medical act.

EHR information can be analysed regarding data type and structure. From a structural perspective, EHRs can contain structured and unstructured data. Structured data can be found in forms, being common in patient demographics data where patient information is organised in form fields. It is also found in certain medical reporting forms, where codes from coding standards such as ICD (International Classification of Diseases) (WHO, 2018), SNOMED-CT (Systematized Nomenclature of Medicine - Clinical Terms) (Stearns et al., 2001) or RxNorm (Nelson et al., 2011) can be used. These standards attempt to structure text data by mapping medical concepts regarding topics such as symptoms, diagnosis, treatments and procedures, to codes which act as unique identifiers and that can be easily processed. However, coding standards also pose certain limitations

Almeida, J., Silva, J., Sierra, A., Matos, S. and Oliveira, J. 
that hinder their use, namely the time required by clinical staff to sift through the standards to select the most adequate code, or the ambiguity or lack of specificity in certain terms.

On the other hand, unstructured data such as free text, is typically present in clinical notes such as clinical appointment reports or patient discharge reports. Since free text is written in natural language, it overcomes the limitations of structured text by providing physicians with a flexible convoy to document complete descriptions of the patient health status, containing the context and rationale for a certain diagnosis or treatment procedure, thus containing valuable information for the processes of clinical decision making and patient follow-up.

Owing to the aforementioned reasons, free text present in clinical notes represents a large amount of the data contained in EHRs. In fact, the importance of free text extends beyond that as it is acknowledged that clinical free text can often encompass information otherwise not obtainable from other data sources (Jensen et al., 2017). However, much of this potential remains underexplored mainly due to the nature of clinical text, which makes it very challenging to process and explore (Neustein et al., 2014). Nonetheless, interest in this domain has shown continuous growth during the past years, with some research efforts having already been made on fields such as clinical natural language processing (NLP) to develop solutions for annotating and summarising relevant data in clinical notes (Pivovarov and Elhadad, 2015).

The increased involvement of technology in healthcare has opened up not only opportunities but also challenges. For instance, the increased availability of medical data, that can aid physicians in their decisions, also resulted in a heavier burden for physicians who must sift through larger amounts of less relevant data to find information of their actual interest. An illustrative example of this can be found in clinical notes, where the increased easiness of replicating information through copy-paste actions has resulted in more redundant information (sometimes even erroneous), and consequently on lower data quality which can ultimately compromise the quality of the medical act (Cohen et al., 2013; Singh et al., 2013).

It comes naturally that solutions such as a clinical decision support system (CDSS) can play an important role by leveraging the large amounts of existing EHR data to provide physicians with only key information. However, when doing so, these systems must deal with the natural challenges associated with medical data, namely its high heterogeneity and poor quality (data is frequently incomplete, noisy and sparse) which are worsened by non-standardized physician practices (Hripcsak and Albers, 2012).

Regarding data sources, these systems can explore single and multi modality - the latter combining various sources and being reckoned as a particularly challenging task (Miotto et al., 2017) - and also structured and/or unstructured data. Structured data is in general more straightforward to exploit, thus being used more frequently. However, the inclusion of unstructured data can provide key content to improve the performance of previously existing systems. For instance, structured text has already been used for various purposes such as prediction modelling (Wu et al., 2010; Ferrão et al., 2013; Ferrão et al., 2016) whereas applications with free text are relatively scarce, despite the acknowledgement that free text holds great potential as a source of relevant data (Jensen et al., 2017).

Progressing from a data to a medical process perspective, clinical practice guidelines (CPGs) consist of systematically developed statements that were created to assist physicians by providing recommendations for diagnosis and treatment guidance (NC$\mathrm{CIH}, 2017)$. Despite the relevance of their objective, CPGs did not have the expected impact on health care, which can be explained by factors such as the lack of time by physicians to learn them, or the fact that CPGs lack manageable workflows that could effectively help putting recommended tasks to practice. EHR-based CDSSs can provide a contribution in treatment guidance, but to succeed it is vital that they incorporate detailed relevant patient data, perform an on the fly evaluation against prior knowledge, and provide recommendations which physicians can act upon (Stewart et al., 2007).

Moreover, to make their impact in health care more significant, CDSSs should be deployed along with slight adjustments in clinic workflow and staff duty, and should be further explored for appointment planning instead of only providing information towards the end of clinical appointments. Several EHRbased CDSSs have already been tested in the past for patients with illnesses such as diabetes, hypertension and others, with the objective of improving key intermediate clinical outcomes of chronic disease care. However, the majority of these systems failed because they missed many of the above-mentioned key aspects (O'Connor et al., 2011).

In this paper, we present a proposal for a system that enriches the health treatment process by automatically extracting information from patient medical notes and aggregating it in clinical protocols, with the goal of leveraging the historical component of the patient trajectory to improve CDSS performance. Our main contributions in this paper are the following: 
- We create new opportunities mainly related to EHR exploration, enabling the enhancement of decision-making processes based on new relationships and pathways between diseases and parental phenotypes;

- The proposed methodology implements different strategies to automatically extract relevant patient clinical information during medical treatments;

- The methodology was integrated in an existing open-source clinical decision support tool to explore newly extracted information in clinical protocols, with the objective of providing better treatment guidance.

\section{GATHERING RELEVANT INFORMATION FROM CLINICAL NOTES}

Clinical notes are an important "tool" for physicians as they keep record of patient trajectories in a readily accessible format, making them suitable for aiding in clinical decision making and patient follow-up. The trajectory component in clinical notes can be particularly evident as these notes can be produced in different stages of health care (e.g. patient admission, discharge, clinical appointment). Despite constituting a big source of relevant patient information (e.g. diagnosis, recommended or followed procedure, medication, family history), many clinical notes are stored and unexplored due to the intricacies of processing free text.

When considering the process of information extraction (IE) from clinical free text, a similar pipeline is used as that explored for common text. This pipeline typically involves two steps: NER (Named Entity Recognition) where entities such as drugs or diseases are identified in the text, and NEN (Named Entity Normalization) where identified entities can be disambiguated and normalised to unique identities. In clinical text, NEN can explore coding standards such as ICD, RxNorm or UMLS (Unified Medical Language System) - a metathesaurus that aggregates multiple lexicons - so as to obtain normalised text which can be stored as structured data. Furthermore, these steps can explore different approaches to process text, namely heuristic and NLP approaches.

Structured text is easier to integrate in clinical decision support systems, therefore it is vital to extract relevant patient information from medical narratives and store it as structured data. Taking that into account, different types of information were extracted from clinical notes. Firstly, heuristics and NLP tech- niques were combined to extract entities related with several classification criteria, namely cardiovascular diseases, medication taken to prevent a given disease, HbA1c values, among others (Antunes. et al., 2019). These criteria were also mapped to possible ICD-9 codes as these enable the augmentation of the dataset with related clinical notes from the MIMIC-III critical care database (Johnson et al., 2016).

Then, focusing on the family history component of clinical notes, a methodology based on heuristics and NLP was used to extract information regarding family members, their association to diseases and living status. For that, clinical text was firstly preprocessed with the Stanford CoreNLP (Manning et al., 2014) dependency parsing and co-reference resolution steps. A lexicon with possible family members was compiled, and the co-reference graph was used along with a set of rules to identify family member mentions. Disease mentions were identified using Neji, a biomedical text annotation server (Matos, 2018), with a disease dictionary compiled from the UMLS. The shortest path in the dependency graph was used to associate disease mentions to family members as well as to determine the living status.

Finally, shifting from NER to NEN tasks, a system was developed targeting clinical concept normalisation. This system uses dictionary matching approaches, with exact and partial matching mechanisms, combined with word embedding similarity to normalise relevant entities in clinical notes. With this approach, identified entities are mapped to their respective concept unique identifier (CUI) from the UMLS metathesaurus.

The above mentioned extraction methodologies were developed under the scope of several research challenges focused on leveraging clinical text, and were validated with datasets from the 2018 n2c2 track on cohort selection for clinical trials, 2019 $\mathrm{n} 2 \mathrm{c} 2 / \mathrm{OHNLP}$ track on family history extraction and 2019 n2c2/OHNLP track on clinical concept normalisation (HMS, 2018; HMS, 2019a; HMS, 2019b).

All relevant patient information resulting from the combined use of these strategies was organised in a data structure ready to be supplied to the CDSS during clinical treatments.

\section{COMBINING PROTOCOLS WITH TEXT DATA}

Clinical guidelines have been created to help health professionals during the treatment of specific pathologies. These guidelines consist of rules and procedures that should be followed during patient evaluation and 
treatment, and can be described in paper format without any digital system. However, due to the number of treatment protocols and their potential complexity, the use of CDSSs is a valuable resource to simplify and optimise health care professionals' tasks.

Therefore, to process clinical guidelines in CDSSs, it is necessary to convert treatment pathways into a digital format. This process can already be performed with existing methodologies such as the Guideline Interchange Format version 3 (GLIF3), which is a model designed to represent shareable computer-interpretable guidelines in the medical field. This model intends to represent different types of guidelines by specifying them following some low-level primitives, which could be applied in screening, diagnosis, and treatment in primary or speciality unit care (Boxwala et al., 2004).

Based on the previous description, we followed a more straightforward approach, as represented in Figure 1. As observable on the left side of Figure 1, the process of converting treatment guidelines into a digital format can be accomplished using only four elements (Almeida et al., 2018). Action elements are responsible for providing treatment recommendations to physicians, which are typically procedures to be executed during treatment. These recommendations are influenced by the Decision elements, which

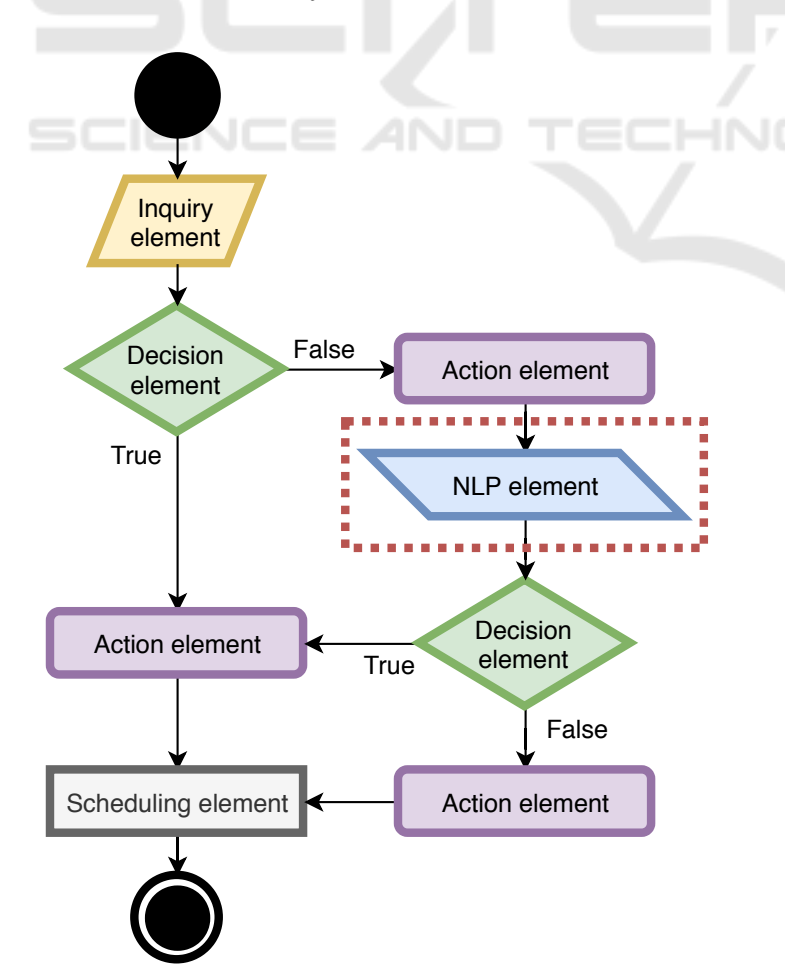

Figure 1: Protocol components for the digital format representation. Surrounded by the red box is the main element for the proposed methodology. are the flow changers in the protocol, mainly because they define the actions that must be done in the treatment based on conditions. Typically, these conditions are constituted by boolean operations that can be represented in the IF-THEN format. Data used in those operations is received from the Inquiry elements that gather patient information before and during treatment. Finally, protocols are executed following a schedule, which is defined using Scheduling elements.

To improve this approach, a fifth protocol element (NLP element) was added that was designed to access information from clinical notes (marked with a red box on the right side of Figure 1). This element identifies information in the patient history that can be relevant to mention when the system gives a treatment recommendation. This type of protocol element also provides suggestions of which would be the information for a specific variable, avoiding inquiry elements. Therefore it simplifies the execution of complex protocols, mainly because it reduces the number of inquiry elements required during treatment.

The use of the NLP element can automatically provide information to the system that is relevant to the treatment. A possible scenario can be that of patients that are taking medication at home and inform the clinical staff during the admission stage about their situation. Commonly, the medications being used and their dosages can have an impact on the treatments that are prescribed. For instance, type 2 diabetic inpatients may need to be medicated daily with insulin dosages, but depending on the insulin product used at home, the treatment in the hospital must be adjusted.

Another scenario is the history of relatives with certain hereditary diseases. With this information, the system can alert the physician to inform the patient about several risks based on their current condition. For instance, patients with irregular blood pressure and diabetic family members are more prone to suffer from diabetes or cardiac diseases in the future. This information can be provided when measurements are done combined with the patient family history.

A final possible scenario concerns protocols for surgery preparation that, depending on the patient state, can lead to a surgery postponement. The system can detect that the patient took a specific medication in past visits and forgot to mention that in a more recent appointment. However, the medication described in their history combined with their current clinical state could indicate that the patient is unable to withstand surgery. 


\section{DECISION SUPPORT TOOL FOR TREATMENT GUIDANCE}

The use of CDSSs for treatment guidance has become an essential piece in continuing care units, as it improves the quality of patient care, helps disease prevention, and supports scientific discoveries. These systems are typically part of the EHR features, some standalone solutions also exist. However, all CDSSs are only able to manage and provide guidance on the medical treatment when clinical protocols are represented in a digital format.

Therefore, in our proposal, we used a nonintegrated CDSS to put the proposed methodology in practice. We used GenericCDSS, an open-source web-based solution that is prepared to create and execute protocols (Almeida and Oliveira, 2019). The tool provides an editor to create and manage clinical protocols in digital format, which we used and improved for our goal, and alerts health professionals about treatment schedules and when the protocol needs to be executed. During protocol execution, the tool requests information about the patient clinical state and then provides several recommendations about the treatment in question.

The typical behaviour of this tool begins by processing the protocol following the workflow structure defined by physicians specialised in a given disease. Then, it considers the clinical information requested during the treatment, and at the end it provides a set of treatment suggestions. The original system input required structured information that could be provided in run-time, however, the same information can be found in the patient diary (in free text) and could be aggregated in the protocol workflow during treatments. Therefore, we created a new feature in the tool to access information extracted from the clinical reports.

These improvements allowed us to assess the impact of enhancing treatment protocols with relevant information from clinical notes on treatment recommendations. We observed that patient clinical state can be complemented with patient history information to obtain better treatments. Therefore, by using the patient history in GenericCDSS, we were able to enrich the protocols and provide more accurate suggestions.

\section{RESULTS AND DISCUSSION}

The previous sections described in detail the different pieces of the proposed methodology. Figure 2 presents the different stages of this workflow. The pipeline starts with the patient admission to the health institution. This is done by a physician after the initial triage when it is defined that the patient will be hospitalised. During patient admission the physician collects patient information such as medication taken at home, family history information and other clinical information relevant to the admission. This data is inserted into the EHR and can be stored in structured format and free-text clinical notes.

After the patient admission and all additional exams are performed, the patient is hospitalised if necessary. In parallel, the system processes the clinical notes to supply the CDSS with additional relevant information. In this stage of the pipeline, notes relevant to the protocol are made available to the CDSS to optimise the process, for when a treatment is required later on.

The next step of the proposed methodology is the treatment guidance supported by the CDSS. Here, the nurse or physician can provide the system with the necessary measurements taken from the patient, which are mostly vital signals and variables that change over time. Clinical information extracted from the admission notes is also provided to the CDSS, reducing considerably the information requested by the system and warning for possible associated risks.

\subsection{Use Case Overview}

The proposed methodology was designed to improve clinical treatments in a general way without specifically following the requirements for a given disease. However, we decided to explore the potential of this proposal in the diabetes scenario. Our main motivation was the lack of effective treatments in health institutions for patients with this disease, mainly due to insufficient exploitation of decision-making systems.

Hyperglycemia is a health condition characterised by abnormally high blood glucose, typically caused by a deficient usage of insulin. Due to the metabolic derangements of this clinical condition, regular monitoring as well as administration of the most effective treatment are major concerns for healthcare institutions. Inpatient hyperglycemia is an event that occurs frequently, with a rate of approximately $40 \%$ of all hospitalisations, rendering it as a metric that deserves special attention from health care institutions and public health services (Inzucchi, 2006).

Basal-bolus insulin therapy is the generally recommended treatment to manage the hyperglycemia of hospitalised diabetic patients (Umpierrez et al., 2012). However, this therapy is also related to high rates of hypoglycemia, reaching values up to $32 \%$, with the main reason for this occurrence being the 


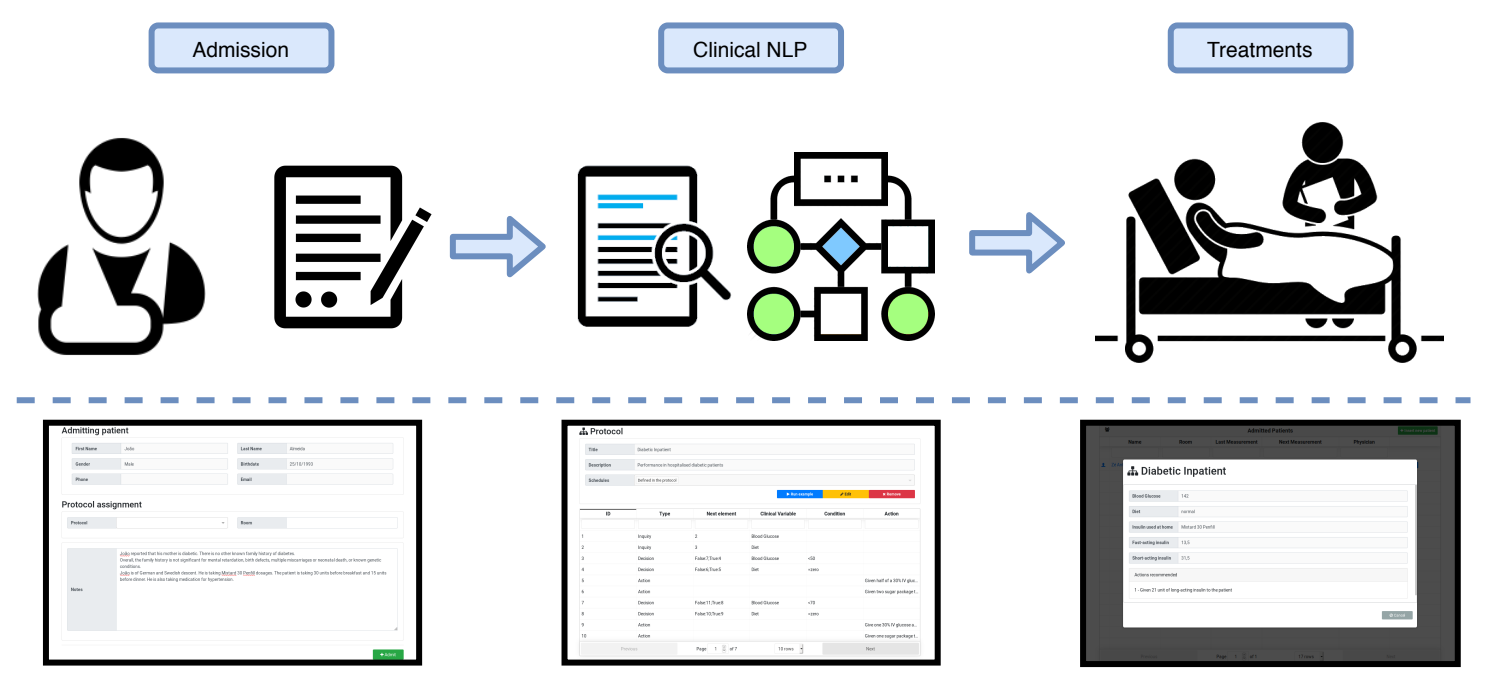

Figure 2: Methodology overview, from admission stage to treatment guidance using the GenericCDSS tool.

meal insulin and food intake mismatch (Umpierrez et al., 2013). As a result, it is possible to recognise that most of the adverse medication occasions and blunders happen when insulin is prescribed or administered. These cases of hypoglycemia in non-intensive care unit settings are a concern because they have been associated with increased length of stay, hospital complications, and mortality (Kim et al., 2014). Several protocols were proposed for glycemic administration to reduce these high rates (Neinstein et al., 2014). However, these procedures are usually available on paper and difficult to follow, which hinders its regular use by non-trained professionals. The goal of the proposed methodology is to reduce this handicap using a system to support the execution of the clinical protocols by considering the information in clinical notes.

\subsection{Protocol Discussion}

Diabetic patients have several base treatments defined depending on their clinical state. Different protocols exist for hospitalised diabetic patients, hypoglycemic patients, diabetic surgical patients, diabetes in pregnant patients, ketoacidosis and hyperosmolar hyperglycemia syndrome in adults and children, among other more specific protocols. However, to describe our methodology more in-depth, we describe the impact of the most common protocol, which is used in hospitalised diabetic patients. This protocol is applied in type 2 diabetic inpatients and has two different stages: the admission moment where the medication taken at home is converted to the one used in the hospital; and then during the patient's stay after the initial set up.
Insulin dosages of patients are defined as Total Daily Dosage (TDD) and calculated based on the patients' information. This value is used as a reference for the basal or long-acting insulin dosages. However, when the patient is taking insulin before the admission, i.e. daily dosages at home, this information must be considered and protocols may need to be changed. Typically, this information is provided during the admission stage and kept in clinical notes, which end up not being adequately considered in the protocols.

Insulin taken by patients at home can contain a mix of long and fast-acting insulin in the same drug, and the percentages of each vary depending on the drug. Therefore, interpreting which drug and dosages are being taken during the day is essential to optimise TDD calculation. Table 1 presents the most common insulin products that patients use at home. LA Insulin and SA Insulin columns represent the percentages of long and short-acting insulin in each product, respectively. With this information, it is possible to determine the total of both insulins taken by the patient

Table 1: Percentage of long (LA) and short-acting (SA) insulins from the most common insulin products used by patients in a domestic setting.

\begin{tabular}{lccc}
\cline { 1 - 1 } Insulin products & & LA Insulin & SA Insulin \\
\cline { 1 - 1 } \cline { 3 - 4 } Mixtard 30 Penfill & & $70 \%$ & $30 \%$ \\
Insuman Comb 25 & & $75 \%$ & $25 \%$ \\
Humulin M3 & & $70 \%$ & $30 \%$ \\
NovoMix 30 & & $70 \%$ & $30 \%$ \\
Humalog Mix 25 & $75 \%$ & $25 \%$ \\
Humalog MIx 50 & $50 \%$ & $50 \%$ \\
\hline
\end{tabular}


and split them as the protocol recommends.

In order to simplify the description of the system execution, the following example is provided:

Example: A patient is taking Mixtard 30 Penfill at home, 30 units before breakfast and 15 units before dinner. This insulin product contains $70 \%$ of long-acting and $30 \%$ of short-acting insulin as it is described in Table 1. Therefore, this patient has a TDD of 45 unit, more precisely 31,5 and 13,5 units of long and short-acting insulin, respectively. Based on the protocol, this patient needs to reduce the total amount of administered insulin, taking only $\frac{2}{3}$ of 31,5 units of long-acting insulin at breakfast during the internment, and $\frac{2}{3}$ of the remaining daily dosage in short-acting insulin.

Moreover, this information is spread over product manuals, patient diaries and clinical guidelines. The system can gather all this information, and following the provided example, it can also recognise that this patient is taking the Mixtard 30 Penfill at home along with the respective dosages. This section describes some relevant aspects that the proposed methodology solves. However, in addition to what has been described, the protocol also considers patient insulin resistance and the different sliding scales present in the protocol must be adjusted depending on patient responses and their plasma glucose values.

\subsection{Validation}

Methodology validation was performed in a controlled environment using a public dataset. The 2014 n2c2 track 2 on de-identification and heart disease risk factors challenge consisted on identifying risk factors for heart disease over time. The dataset used in this challenge contained 1,304 clinical narratives from 296 diabetic patients (2-5 records per patient). These narratives contain information about heart disease risk factors such as high blood pressure and cholesterol levels (Stubbs et al., 2015).

Therefore, we selected 25 patients whose notes contained more information to create some difficulties in the NLP methodologies (e.g. more redundant and less concise information). Additionally, we randomly added sentences indicating that the patient is taking insulin products at home, and following some criteria, we also added information about dosages that they administered during the day (before breakfast, lunch, dinner, meals or bedtime).

Then, we manually simulated the physician work and protocol execution during treatments. Altogether, the proposed methodology produced positive results.
However, we noticed that the system faced some complications in the clinical notes analysis stage. The system had issues when randomly inserted sentences were too complex, referencing past medication that is currently not being taken by the patient. However, we solved this issue by giving the possibility to the physician to consult the clinical report in run-time, identifying which were the sentences that originated that recommendation.

\section{CONCLUSION}

The secondary use of clinical notes is a subject that has been under study over the past years. In the medical field, the use of systems for decision-making and treatment guidance is a subject of much research. Moreover, we detected an opportunity to enrich medical treatments by combining both topics with the objective of reducing existing gaps in treatment prescriptions.

The proposed methodology was integrated and validated with an open-source CDSS due to its autonomy and ease of development. However, the goal was to demonstrate the positive impact of combining these subjects and define a supportive approach. The methodology can be applied in the different decisionmaking features existent in the EHR systems available in the market.

As future work, and to better understand possible improvements, we expect to apply this methodology with different diseases beyond diabetes. Additionally, to validate the protocol recommendations, as well as the extracted clinical concepts, we intend to incorporate a classification feature. With this feature, the physician can evaluate the accuracy of the system, which may thus help to increase the performance and discover possible gaps to be addressed.

\section{ACKNOWLEDGEMENTS}

This work has received support from the EU/EFPIA Innovative Medicines Initiative 2 Joint Undertaking under grant agreement No 806968 and from the NETDIAMOND project (POCI-01-0145-FEDER016385), co-funded by Centro 2020 program, Portugal 2020, European Union. João Figueira Silva and João Rafael Almeida are funded by the FCT - Foundation for Science and Technology (national funds) under the grants $\mathrm{PD} / \mathrm{BD} / 142878 / 2018$ and SFRH/BD/147837/2019 respectively. 


\section{REFERENCES}

Almeida, J. R., Guimarães, J., and Oliveira, J. L. (2018). Simplifying the digitization of clinical protocols for diabetes management. In 2018 IEEE 31st International Symposium on Computer-Based Medical Systems (CBMS), pages 176-181. IEEE.

Almeida, J. R. and Oliveira, J. L. (2019). GenericCDSSa generic clinical decision support system. In 2019 IEEE 32nd International Symposium on ComputerBased Medical Systems (CBMS), pages 186-191. IEEE.

Antunes., R., Silva., J. F., Pereira., A., and Matos., S. (2019). Rule-based and machine learning hybrid system for patient cohort selection. In Proceedings of the 12th International Joint Conference on Biomedical Engineering Systems and Technologies - Volume 2: HEALTHINF, pages 59-67. INSTICC, SciTePress.

Boxwala, A. A., Peleg, M., Tu, S., Ogunyemi, O., Zeng, Q. T., Wang, D., Patel, V. L., Greenes, R. A., and Shortliffe, E. H. (2004). Glif3: a representation format for sharable computer-interpretable clinical practice guidelines. Journal of biomedical informatics, 37(3):147-161.

Cohen, R., Elhadad, M., and Elhadad, N. (2013). Redundancy in electronic health record corpora: analysis, impact on text mining performance and mitigation strategies. BMC Bioinformatics, 14(10).

Costa, C. M. A. (2004). Concepção, desenvolvimento e avaliação de um modelo integrado de acesso a registos clínicos electrónicos. PhD thesis, University of Aveiro.

Ferrão, J. C., Janela, F., Oliveira, M. D., and Martins, H. M. G. (2013). Using structured EHR data and SVM to support ICD-9-CM coding. In 2013 IEEE International Conference on Healthcare Informatics, pages 511-516, Philadelphia, PA, USA. IEEE.

Ferrão, J. C., Oliveira, M. D., Janela, F., and Martins, H. M. G. (2016). Preprocessing structured clinical data for predictive modeling and decision support. Applied Clinical Informatics, 07(04):1135-1153.

HMS (2018). 2018 n2c2 - Track 1: Cohort Selection for Clinical Trials.

HMS (2019a). 2019 n2c2 Shared-Task and Workshop, Track2: n2c2/OHNLP Track on Family History Extraction.

HMS (2019b). 2019 n2c2 Shared-Task and Workshop, Track3: n2c2/UMass Track on Clinical Concept Normalization.

Hripcsak, G. and Albers, D. J. (2012). Next-generation phenotyping of electronic health records. Journal of the American Medical Informatics Association, 20(1):117-121.

Inzucchi, S. E. (2006). Management of hyperglycemia in the hospital setting. New England journal of medicine, 355(18):1903-1911.

Jensen, K., Soguero-Ruiz, C., Oyvind Mikalsen, K., Lindsetmo, R.-O., Kouskoumvekaki, I., Girolami, M., Olav Skrovseth, S., and Augestad, K. M. (2017). Analysis of free text in electronic health records for identification of cancer patient trajectories. Scientific Reports, 7(46226)

Johnson, A. E. W., Pollard, T. J., Shen, L., Lehman, L.w. H., Feng, M., Ghassemi, M., Moody, B., Szolovits, P., Anthony Celi, L., and Mark, R. G. (2016). MIMICIII, a freely accessible critical care database. Scientific Data, 3

Katehakis, D. G. and Tsiknakis, M. (2006). Electronic health record. In Wiley Encyclopedia of Biomedical Engineering. Wiley.

Kim, Y., Rajan, K. B., Sims, S. A., Wroblewski, K. E., and Reutrakul, S. (2014). Impact of glycemic variability and hypoglycemia on adverse hospital outcomes in non-critically ill patients. Diabetes research and clinical practice, 103(3):437-443.

Manning, C. D., Surdeanu, M., Bauer, J., Finkel, J., Bethard, S. J., and McClosky, D. (2014). The Stanford CoreNLP natural language processing toolkit. In Association for Computational Linguistics (ACL) System Demonstrations, pages 55-60.

Matos, S. (2018). Configurable web-services for biomedical document annotation. Journal of cheminformatics, 10(1):68.

Miotto, R., Wang, F., Wang, S., Jiang, X., and Dudley, J. T. (2017). Deep learning for healthcare: review, opportunities and challenges. Briefings in Bioinformatics.

NCCIH (2017). Clinical Practice Guidelines.

Neinstein, A., MacMaster, H. W., Sullivan, M. M., and Rushakoff, R. (2014). A detailed description of the implementation of inpatient insulin orders with a commercial electronic health record system. Journal of diabetes science and technology, 8(4):641-651.

Nelson, S. J., Zeng, K., Kilbourne, J., Powell, T., and Moore, R. (2011). Normalized names for clinical drugs: RxNorm at 6 years. Journal of the American Medical Informatics Association, 18(4):441.

Neustein, A., Imambi, S. S., Rodrigues, M., Teixeira, A., and Ferreira, L. (2014). Application of text mining to biomedical knowledge extraction: analyzing clinical narratives and medical literature. In Text Mining of Web-based Medical Content, pages 3-32. De Gruyter.

O'Connor, P. J., Sperl-Hillen, J. M., Rush, W. A., Johnson, P. E., Amundson, G. H., Asche, S. E., Ekstrom, H. L., and Gilmer, T. P. (2011). Impact of electronic health record clinical decision support on diabetes care: a randomized trial. Annals of family medicine, 9(1):1221.

Pivovarov, R. and Elhadad, N. (2015). Automated methods for the summarization of electronic health records. Journal of the American Medical Informatics Association, 22(5):938-947.

Singh, H., Giardina, T. D., Meyer, A. N. D., Forjuoh, S. N., Reis, M. D., and Thomas, E. J. (2013). Types and origins of diagnostic errors in primary care settings. JAMA Internal Medicine, 173(6):418-425.

Stearns, M. Q., Price, C., Kent A. Spackman, and Wang, A. Y. (2001). SNOMED clinical terms: overview of the development process and project status. In Proceedings of the AMIA Symposium, pages 662-666, 
Washington, DC, USA. American Medical Informatics Association.

Stewart, W. F., Shah, N. R., Selna, M. J., Paulus, R. A., and Walker, J. M. (2007). Bridging the inferential gap: The electronic health record and clinical evidence. Health Affairs, 26(Supplement 1):w181-w191.

Stubbs, A., Kotfila, C., Xu, H., and Uzuner, Ö. (2015). Identifying risk factors for heart disease over time: Overview of 2014 i2b2/uthealth shared task track 2. Journal of biomedical informatics, 58:S67-S77.

Umpierrez, G. E., Gianchandani, R., Smiley, D., Jacobs, S., Wesorick, D. H., Newton, C., Farrokhi, F., Peng, L., Reyes, D., Lathkar-Pradhan, S., et al. (2013). Safety and efficacy of sitagliptin therapy for the inpatient management of general medicine and surgery patients with type 2 diabetes: a pilot, randomized, controlled study. Diabetes Care, 36(11):3430-3435.

Umpierrez, G. E., Hellman, R., Korytkowski, M. T., Kosiborod, M., Maynard, G. A., Montori, V. M., Seley, J. J., and Van den Berghe, G. (2012). Management of hyperglycemia in hospitalized patients in non-critical care setting: an endocrine society clinical practice guideline. The Journal of Clinical Endocrinology \& Metabolism, 97(1):16-38.

WHO (2018). World Health Organization: International classification of diseases, 11th Revision (ICD-11).

Wu, J., Roy, J., and Stewart, W. F. (2010). Prediction modeling using EHR data: challenges, strategies, and a comparison of machine learning approaches. Medical Care, 48(6):S106-S113. 